\title{
Implementing motivational interviewing training: Strengthening the role of the registered nurse
}

\author{
Alicia Russell Maloney *1, Linda Ehrlich-Jones ${ }^{2,3}$ \\ ${ }^{1}$ Department of Biobehavioral Health Science, University of Illinois at Chicago, Chicago, Illinois, United States \\ ${ }^{2}$ Rehabilitation Institute of Chicago, Chicago, Illinois, United States \\ ${ }^{3}$ Department of Physical Medicine \& Rehabilitation, Feinberg School of Medicine, Northwestern University, Chicago, Illinois, \\ United States
}

Received: February 6, 2017

Accepted: March 1, 2017

Online Published: March 9, 2017

DOI: $10.5430 /$ jnep.v7n8p51

URL: https://doi.org/10.5430/jnep.v7n8p51

\begin{abstract}
The current primary healthcare model is in need of urgent transformation, as it is vital to addressing health priorities at the systemic level. With over 3 million members, the nursing profession is the largest entity of the nation's healthcare workforce, however they are underutilized in the primary care setting. As the pendulum swings back towards community based primary care, changes in nursing education are critical for support. Nursing curricula needs to be reexamined, updated, and adaptive enough to change with patients' needs and improvements in both science and technology. The Josiah Macy Jr. Foundation has identified and responded to these needs with specific themes and recommendations on how best to prepare the registered nurse in the enhanced team member role. The purpose of this article, is to further explore the education of nursing students with a focus in primary care and consider the use of motivational interviewing, an evidenced-based intervention, to bridge a gap in nursing curricula. Faculty members are in a strategic position to educate and introduce the nursing future about the use of motivational interviewing, to help patients make healthier choices and impact future health outcomes.
\end{abstract}

Key Words: Motivational Interviewing (MI), Nursing curriculum, Behavior change, Primary healthcare

\section{INTRODUCTION}

Both primary and public healthcare are vital to addressing health priorities at the systemic level; however, the current model is in need of urgent transformation. ${ }^{[1,2]}$ The 2010 Affordable Care Act (ACA) seeks to transform the healthcare system to provide millions of Americans the capacity to seek routine, accessible and affordable care. Necessary resources to meet these goals are inadequately allocated and a comprehensive rethinking of the roles of many healthcare professionals is warranted. With over 3 million members, the nursing profession is the largest entity of the nation's healthcare workforce, however they are underutilized in the primary care setting. ${ }^{[2,3]}$ Who better to help expand and build the primary care capacity on the front line of patient care? After the 2011 release of The Institute of Medicine's Future of Nursing report, significant progress has been made concerning the role of the advanced practice nurse; however, little attention has been paid to the report's implication for registered nurses in transforming primary care to address the needs of the nation. ${ }^{[3,4]}$ The American Academy of Nursing approached the Macy Foundation to raise the significance of this issue. The Macy Foundation has since responded

\footnotetext{
*Correspondence: Alicia Russell Maloney; Email: arusse6@uic.edu; Address: Department of Biobehavioral Health Science, University of Illinois at Chicago, Chicago, Illinois, United States.
}

Published by Sciedu Press 
with specific themes and recommendations on how best to prepare the registered nurse in the enhanced team member role. The purpose of this article, is to further explore the education of undergraduate nursing students with a focus in primary care and consider the use of motivational interviewing, an evidenced-based intervention, to bridge a gap in nursing curricula.

\section{BACKGROUND}

Eating healthy, exercising regularly, avoiding tobacco, and receiving preventive services such as cancer screenings and vaccinations are all examples of the ways in which individuals can maintain their health. Yet, as a nation, preventive services are used at only half the recommended rate. ${ }^{[5]}$ This finding highlights the need for health promotion and disease prevention. There is an increasing focus on the management of chronic conditions, which speaks to the growing need to promote lifestyle changes, which modify risk factors. ${ }^{[6,7]}$

Motivational interviewing (MI) is a method for encouraging people to make behavioral changes to improve health outcomes. ${ }^{[8]} \mathrm{MI}$, as a strategy to promote behavior change, historically has its roots in the addictions field. MI is utilized as a goal-oriented counseling approach, which is clientcentered for eliciting behavior change by helping patients to explore and resolve ambivalence. MI is based on four key processes: 1) therapeutic engagement, 2) focusing on an agenda, 3) evoking change, and 4) making a plan of action. The spirit of MI encompasses a collaborative process, using the ideas of the client to autonomously evoke change and proceed when ready. ${ }^{[9]}$

In more recent years, MI has progressively been tested and applied to areas of other health problems such as management of cardiovascular disease, diabetes, weight loss, hypertension and prevention of HIV infection, to name a few. ${ }^{[8]}$ $\mathrm{MI}$ is flexible, in that it produces desirable outcomes in problem areas across the primary care field; one valuable aspect, is emphasis placed on the training of healthcare providers from various backgrounds. ${ }^{[6,10]}$ There are specific aspects of communication one must learn to become proficient in MI such as reflective listening, formulating open-ended questions and affirming the client. ${ }^{[7,9]} \mathrm{MI}$ is appealing to primary care because it can be viewed as a practical front line intervention, which reflects the overarching goals of the national healthcare strategy. ${ }^{[11]}$

The ACA aims to elevate prevention and chronic care management to a national priority, providing opportunities for health promotion through all policies, which will build on the foundation of preceding initiatives, such as Healthy People 2020. ${ }^{[12]}$ Motivational interviewing is just one specific example of a way to progress from advice-giving roles to a client-centered counseling method. ${ }^{[9]}$ To ensure efforts are successful, national needs are shifting primary care towards new practice models staffed by high-functioning, interprofessional teams. ${ }^{[3,13]}$ While the large nursing workforce has the potential to help meet the demands facing primary care today, a number of barriers must be overcome. ${ }^{[3]}$ The Macy Foundation ${ }^{[3]}$ reports the most important hurdle as inconsistency to the full range of primary care content received in the classroom or through instructional clinical experiences, which focus heavily on inpatient and acute care. As a result, these nurses may lack the skills and competencies essential to functioning as an effective member of the interprofessional primary care team. ${ }^{[3]}$

As the pendulum swings back towards community based primary care, changes in nursing education are critical for support. One actionable recommendation made to support the education of nursing students in primary care reads:

"Nursing faculty must broaden and deepen the primary care focus in the curriculum. Doing so includes enriching content on topics such as wellness, health promotion, and disease prevention; population health and risk stratification; motivational interviewing and health coaching; health equity; leadership, cost of care, delivery models and systems innovations... ${ }^{[3]}$ ",

Nursing school leaders and faculty should use a multipronged approach to elevate primary care content, in the education of nursing students. The recommendation has been made to rebalance the curricula, to incorporate adult learning theory and educational scholarship, between acute and primary care instruction and furthermore, support the graduate interested in seeking a primary care role in their nursing career. ${ }^{[3]}$

\section{LITERATURE REVIEW}

Medical practice settings that have utilized registered nurses in enhanced roles have shown improved health outcomes, reduction in costs and enhanced patient satisfaction. ${ }^{[3]}$ The way in which health is discussed with patients greatly influences their personal motivation for behavior change. ${ }^{[8]}$ In an effort to delve into current nursing curriculum issues, as it applies directly to primary care, this literature review will focus on motivational interviewing as a nursing skill to enhance patient outcomes.

Motivational interviewing is an evidence-based intervention, used in a wide range of health behaviors. In a metaanalysis conducted by Rubak, Sandbaek, Lauritzen and Christensen, ${ }^{[14]} 64 \%$ of the 72 randomized controlled trails analyzed showed a positive effect after only one brief MI 
encounter. VanBuskirk and Wetherell ${ }^{[15]}$ conducted a metaanalysis and found MI to be effective, in comparison to usual care, in as little as one 15 to 20 minute session. This success was also pertinent to MI delivery techniques such as telephone intervention or when "boosted" by intermittent phone calls after the initial in-person session. In summary, MI was found to be flexible in both its formulation and delivery within the primary care population.

The majority of MI studies are focused outside of the primary care setting. The first meta-analysis and systematic review to focus on MI efficacy in the primary care setting was conducted by Lundahl et al. ${ }^{[6]}$ When looking to MI as a potential treatment intervention, $63 \%$ of the main outcome comparisons show MI to have beneficial effects. It can be successfully delivered by an array of medical personnel, in a variety of settings, formats and time frames to service differing ethnicities, ages and genders alike. Furthermore, primary care providers can utilize MI to assist patients in weight loss, increased exercise, control of cholesterol and blood pressure and reduction of substance abuse, all while boosting self-efficacy in their health related behavior change abilities.

Registered dietician nutritionists (RDNs) have found MI useful in improving behaviors surrounding fruit and vegetable consumption, increasing physical activity and short-term weight loss. ${ }^{[7]}$ Although MI has gained popularity among the RDN discipline, Smart et al. ${ }^{[7]}$ reports adequate training is lacking and a structured program would benefit the undergraduate dietician students. A small quasi- experimental pilot study took a closer look into MI curricula, grounded in the Adult Learning Theory (ALT). Both student-learning groups used classmates as standardized patients to conduct a 30-minute videotaped counseling sessions. Prior to the session, each group participated in 300 minutes of instructed MI curriculum. The intervention group received the MI curriculum prior to the counseling sessions and before the post surveys were complete. The curriculum included lectures, videos, worksheets, case studies and role-playing activities. ALT focuses on the concept in that adults prefer to learn skill application versus abstract theory; therefore, the focus was on application of MI skills in counseling. The 300-minute ALT based curriculum on MI knowledge, skills and counseling was found to be effective, as evidenced by the increase in MI knowledge after the educational experience and compared to the intervention group. The initial MI exposure to training of future practitioners should be considered a foundation for undergraduate curriculum; MI is a cornerstone to the preparation of students for internships and expanded roles in primary care.
Another quasi-experimental pilot study, conducted by Nesbitt et al. ${ }^{[16]}$ examined counseling skill outcomes of a brief educational module for graduate nursing students. Core competencies for the graduate nursing student speak to the education surrounding the adoption of positive health behavior change, however little has been published as to how to incorporate teaching the MI skill set within the curriculum. The intervention consisted of a brief educational module consisting of a four-week exposure to MI utilizing class lecture, discussion, videotaped practice, experiential application and independent readings.

Martino, Haeseler, Belitsky, Pantalon, and Fortin ${ }^{[17]}$ developed and tested a curriculum for third year medical students called brief motivational interviewing (BMI). Despite time constraints seen in the modern day medical practice, this curriculum was designed to promote behavior change in patients. To promote the student learning aspect, both instructors and standardized patients delivered the one-time, 2-hour training session using a teaching acronym called CHANGE. A pretest, posttest and 4 week follow up design was followed to assess BMI skill level, knowledge and attitude towards the intervention. The students were able to successfully increase the depth and frequency of their patient reflections and reduced the frequency in which they used closed ended responses. BMI knowledge was increased, as well as their confidence in MI delivery and overall interest in the intervention.

In a focused ethnography by Howard and Williams, ${ }^{[18]}$ they describe how nursing students in a community-based primary care vascular risk reduction clinic, learned and used motivational interviewing. The aim was to provide assistance to nurse educators on how to better prepare their students with patients working on health change behaviors. A central finding highlights the student experience of learning and the application of MI, which in turn transformed their roles as health care professionals. A common theme was the timing and introduction of the MI skill in their academic curriculum; the students' reported this as a key to their success. At this point in their academic career, the student is expanding beyond the hands-on clinical skill set and focusing more on relational skills. To enhance the MI skill development, instructors assisted by providing ongoing feedback. This feedback focused on patient engagement and the use of critical thinking to tailor health messages. Instructional feedback sessions are an opportunity to engage in student self-reflection and recognize areas for improvement. These findings further reflect the current recommendations on MI teaching/learning that performance assessment and feedback from a skilled professional after that initial exposure is necessary for the continued student skill development of the student. ${ }^{[15,18]}$ 
In summary, motivational interviewing is a potential cost effective intervention that can be implemented in as little as 15 minutes, to positively influence the behavior change desperately needed in the primary care area. MI has been the focus of many research endeavors, explored in numerous medical settings and formats. The Adult Learning Theory conceptualizes the practicality surrounding MI, as nursing students prefer the application of a skill, as opposed to abstract theory. The question that has not yet been answered, as we move forward is how best to incorporate both the didactic and clinical learning components of MI into the nursing curricula. One additional key component is the use of trained educators, again in the classroom and clinical settings, to introduce and enhance this newly learned skill.

\section{INTERVENTION APPLICATIONS}

After reviewing the literature, it is clear that motivational interviewing has a profound role in the care of the primary care patient. The bar has been set by the Josiah Macy Jr. Foundation and The Institute of Medicine, which recommends that nurses, nursing students and faculty continue their education and engage in lifelong learning. So how to best teach our nursing students to utilize the spirit, principles and skills of motivational interviewing from their first academic exposure to last through a successful career? Continued lifelong learning is a foundational platform in which nursing education stands; exposure to MI in undergraduate education is an important opportunity to embed this skill into routine nursing care. $^{[18]}$

Howard and Williams ${ }^{[8]}$ touch upon one of the most prudent points, as to when to introduce this content into the undergraduate nursing education timeline. The most effective learning time is when the student is beyond the hands-on skill set and into the critical thinking and relational aspects of their academic careers. The amount of time that needs devoted to training has varied, yet outcomes have been positive. ${ }^{[16]}$ The literature reports one 300 minute training session effective, whereas another didactic approach, known as Group Motivational Interviewing (GMI), is another focused competency alternative. ${ }^{[7,18]}$ GMI facilitates active participation and exploration of personal goals, which is the foundational principal of MI in client-centered counseling. Booster training sessions are recommended to maintain skills, continue learning and increase confidence with MI application. ${ }^{[16]}$ It is unclear when and where these booster-training sessions are to take place and at what time intervals? Further research is needed to report on time frequency and the importance of booster sessions.

Emerging new competencies, such as MI in decision-making, quality improvement, systems thinking, and team leadership must become part of every nurse's professional formation. ${ }^{[4]}$ Innovative and unique teaching/learning opportunities have emerged from the literature on how best to incorporate MI learning into curriculum. Effective teaching and educational methods such as independent study, workshop style presentations, audio and video taped role-play, and the use of outside consultants have been reported successful in the literature. The use of high fidelity simulation has also been reported as a proficient and feasible strategy for teaching MI to nursing students, surrounding basic techniques and principles. ${ }^{[19]}$ The main take away concept should incorporate practicing with real patients and real time experience. The one-on-one clinical experience, paired with ongoing and personalized feedback from a skilled instructor, is imperative to the proficiency of the nursing student with MI. ${ }^{[18]}$

\section{Discussion}

Those patients who received MI had one and a half times the chance of improving on a multitude of health measures. ${ }^{[6]}$ No matter your professional training or setting of work, if you can build relationships and evoke change talk through a small amount of devoted extra time, this could be the transformation the primary care area desperately needs, in a variety of behavioral outcomes. The need exists to pair this knowledge base with the three aims of The Macy Foundation, ${ }^{[3]}$ including: to successfully strengthen the core of primary care services with improved patient care experiences, better population health outcomes and lower health costs. In other words, how can undergraduate nursing students become proficient with motivational interviewing, in an effort to strengthen the primary care quality improvement infrastructure?

Nursing curricula needs to be reexamined, updated, and adaptive enough to change with patients' needs and improvements in both science and technology. Integrating MI into nursing curriculum is an important first step of this evidence-based approach, effecting positive behavior change. The ideal place to develop MI proficiency is the clinical setting. ${ }^{[9,16]}$ Verbal feedback, in real time, is an instrumental step in the application of MI skill and spirit. This allows the students to engage patients in conversations about their health, tailor information to their needs and engage in a collaborative partnership for the exploration of their health behavior change. ${ }^{[15,18]}$

Although the one-on-one experience is ideal for proficiency, a didactic approach is the initial exposure point for the novice. From a feasibility standpoint, it may not be possible for all nursing programs to provide proficient training in MI, however 300 minutes is at least sufficient for foundational knowledge. ${ }^{[7]}$ Curriculum committees in academic settings can decide where to place the didactic course material, prior to the clinical learning experience. The Adult Learning Theory 
is just one possibility to ground didactic curriculum efforts moving forward, however more literature is needed on course content building. From the students' perspective, year three of their curriculum was an ideal time to learn MI, as they expand upon relational techniques alongside hands-on care. ${ }^{[18]}$ At present, there are no studies to report from the educators' perspective on the most valuable, realistic and capable timeframe for the undergraduate nursing student to learn and incorporate MI.

Faculty members are in a strategic position to educate and introduce the nursing future about the use of MI to help patients make healthier choices and impact future health outcomes. ${ }^{[16]}$ More prepared faculty are needed who are trained in MI. The Motivational Interviewing Network of Trainers (MINT), is a solid example of a resource educators can use to build upon and share their experience with MI. ${ }^{[20]}$ Instructors should be sensitive to the timing of MI introduction into curriculum and establish clear linkages for students to the role MI has in enhancing nursing care. ${ }^{[18]} \mathrm{To}$ build upon the foundational aspects of MI, some suggestions moving forward include the addition of internships, clinical practicum experience, enhanced direct supervision and continuing education.

\section{Conclusions}

The ways in which nurses were educated during the 20th century are no longer adequate for dealing with the realities of the chronic health issues we now face in the 21 st century. Motivational interviewing may have a profound place in primary care, as a quality improvement intervention to meet the healthcare needs of our growing nation. The goal is to improve patient and provider quality experiences, with reduction in costs. As the role of the registered nurse expands, evolves and collaborates, the quality improvement capacity of primary care builds. The experience of engaging patients through MI and collaborating as partners in their health goals enables the student to demonstrate critical thinking skills and transform their effect on future practice. ${ }^{[18]}$ If these techniques are capable of generalization, it is possible that these training approaches will be a significant resource to addressing the multiple behavior change difficulties that are affecting the average modern primary care patient. ${ }^{[15]}$ To translate these ideas into reality, the work must begin at the academic level. Further work is needed to understand the feasibility of adding MI to the already stringent undergraduate nursing curriculum; however, nursing organizations around the country must rise to the occasion and equip our future leaders with the competencies of health policy, system improvement, research and evidence-based practice, teamwork and collaboration.

\section{Conflicts of InTEREST Disclosure}

The authors declare that there is no conflict of interest.

\section{REFERENCES}

[1] CDC. Primary care and public health initiative. 2016. Available from: http://www.cdc.gov/ophss/csels/dsepd/academic - partnerships/primary-care.html

[2] Institute of Medicine. The future of nursing: Focus on education. (Report Brief). 500 Fifth Street NW Washington, DC 20001: National Academy of Sciences. 2010

[3] Josiah Macy Jr. Foundation. Registered nurses: Partners in transforming primary care. (Conference Recommendations No. June 15-18, 2016). 44 East 64th Street, New York, NY 10065. (Recommendations from the Macy Foundation Conference on Preparing Registered Nurses for Enhanced Roles in Primary Care). 2016.

[4] Institute of Medicine. The future of nursing: Leading change, advancing health. Washington, DC: The National Academies Press; 2011.

[5] CDC. Gateway to health communication \& social marketing practice: Preventive health care. Available from: http://www.cdc.gov/healthcommunication/ToolsTempl ates/EntertainmentEd/Tips/PreventiveHealth.html

[6] Lundahl B, Moleni T, Burke BL, et al. Motivational interviewing in medical care settings: A systematic review and meta-analysis of randomized controlled trials. Patient Education and Counseling. 2013; 93(2): 157-168. https://doi.org/10.1016/j.pec. 2013 .07 .012

Published by Sciedu Press
[7] Smart H, Clifford D, Morris MN. Nutrition students gain skills from motivational interviewing curriculum. Journal of the Academy of Nutrition and Dietetics. 2014; 114(11): 1712-1717. https: //doi.org/10.1016/j.jand.2014.04.012

[8] Rollnick S, Miller WR, Butler CC. Motivational interviewing in health care: Helping patients change behavior. New York, NY: The Guilford Press; 2008.

[9] Miller WR, Rollnick S. Motivational interviewing: Helping people change (3rd ed.). New York, NY: Guilford Press; 2013.

[10] Madson MB, Loignon AC, Lane C. Training in motivational interviewing: A systematic review. Journal of Substance Abuse Treatment. 2009; 36(1): 101-109. https://doi.org/10.1016/j.jsat. 200 8.05 .005

[11] Coyne N, Correnti D. Effectiveness of motivational interviewing to improve chronic condition self-management: What does the research show us? Home Healthcare Nurse. 2014; 32(1). https: //doi.org/10.1097/NHH.0000000000000001

[12] Koh KK. A 2020 vision for healthy people. The New England Journal of Medicine. 2010; 362: 1653-1656. https://doi.org/10.105 6/NEJMp1001601

[13] Public Law. In 42 USC 18001 (Ed.), The patient protection and affordable care act (111-148 ed.) 111th Congress. 2010. 
[14] Rubak S, Sandbaek A, Lauritzen T, et al. Motivational interviewing: A systematic review and meta-analysis. The British Journal of General Practice: The Journal of the Royal College of General Practitioners. 2005; 55(513): 305-312. PMid:15826439

[15] VanBuskirk KA, Wetherell JL. Motivational interviewing with primary care populations: A systematic review and meta-analysis. Journal of Behavioral Medicine. 2014; 37(4): 768-780. https: //doi.org/10.1007/s10865-013-9527-4

[16] Nesbitt BJ, Murray DA, Mensink AR. Teaching motivational interviewing to nurse practitioner students: A pilot study. Journal of the American Association of Nurse Practitioners. 2014; 26(3): 131-135. https ://doi.org/10.1002/2327-6924.12041

[17] Martino S, Haeseler F, Belitsky R, et al. Teaching brief motivational interviewing to year three medical students. Medical Education. 2007;
41(2): 160-167. PMid:17269949 https://doi.org/10.1111/j. 1365-2929.2006.02673.x

[18] Howard LM, Williams BA. A focused ethnography of baccalaureate nursing students who are using motivational interviewing. Journal of Nursing Scholarship: An Official Publication of Sigma Theta Tau International Honor Society of Nursing. 2016; 48(5): 472-481. https://doi.org/10.1111/jnu.12224

[19] Czart M. Using 3D standardized patients to teach motivational interviewing: A pilot study. Journal of Virtual Worlds Research. 2014; 7(2): 1-24.

[20] Miller B, Rollnick S. Motivational interviewing network of trainers. 2016. Available from: http://www.motivationalinterviewi ng.org/about_mint 